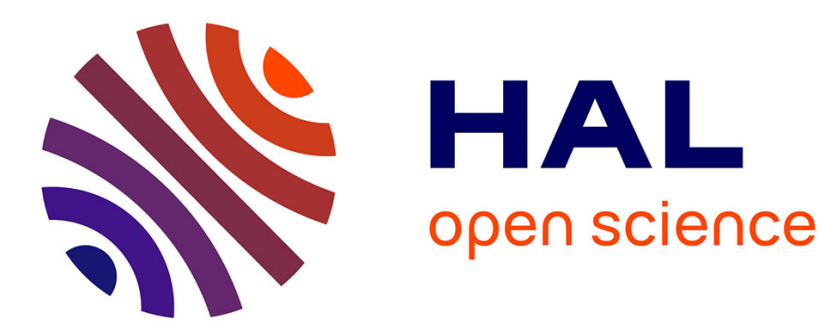

\title{
Amélioration du taux d'ondulation d'un générateur haute tension pour un canon à émission de champ
}

M. Troyon, E. Merienne

\section{To cite this version:}

M. Troyon, E. Merienne. Amélioration du taux d'ondulation d'un générateur haute tension pour un canon à émission de champ. Revue de Physique Appliquée, 1974, 9 (3), pp.533-537. 10.1051/rphysap:0197400903053300 . jpa-00243814

HAL Id: jpa-00243814

https://hal.science/jpa-00243814

Submitted on 1 Jan 1974

HAL is a multi-disciplinary open access archive for the deposit and dissemination of scientific research documents, whether they are published or not. The documents may come from teaching and research institutions in France or abroad, or from public or private research centers.
L'archive ouverte pluridisciplinaire HAL, est destinée au dépôt et à la diffusion de documents scientifiques de niveau recherche, publiés ou non, émanant des établissements d'enseignement et de recherche français ou étrangers, des laboratoires publics ou privés. 
Classification

Physics Abstracts

0.691

\title{
AMÉLIORATION DU TAUX D'ONDULATION D'UN GÉNÉRATEUR HAUTE TENSION POUR UN CANON A ÉMISSION DE CHAMP
}

\author{
M. TROYON et E. MERIENNE
}

\author{
Laboratoire d'Electricité et de Microscopie Electronique $(*)$ \\ Faculté des Sciences, 51062 Reims Cedex, France
}

(Reçu le 13 novembre 1973, révisé le 12 décembre 1973)

\begin{abstract}
Résumé. - Nous décrivons un dispositif très économique qui permet d'obtenir un taux d'ondulation de l'ordre de $5 \times 10^{-7}$ dans les conditions normales d'utilisation du microscope électronique avec une cathode à émission de champ et inférieur à $1 \times 10^{-7}$ en utilisation avec une cathode thermoionique.
\end{abstract}

\begin{abstract}
A very inexpensive device is described that allows us to obtain a relative voltage ripple of about $5 \times 10^{-7}$ in the normal working conditions of an electron microscope using a field emission gun and better than $1 \times 10^{-7}$ using a standard gun.
\end{abstract}

1. Introduction. - Pour atteindre de très hautes résolutions de l'ordre de $1 \AA$ en microscopie électronique par transmission, une alimentation haute tension très stable est nécessaire. Une étude récente [1] de l'amortissement des fonctions de transfert du contraste de phase, dû aux oscillations sinusoïdales des alimentations, montre que le taux d'ondulation nécessaire à l'obtention d'une résolution de $1 \AA$, avec une tension d'accélération de $100 \mathrm{kV}$ et un coefficient d'aberration chromatique $C_{\mathrm{c}}=2 \mathrm{~mm}$, est de l'ordre de $5 \times 10^{-7}$.

Un des objectifs de notre laboratoire pour atteindre les très hautes résolutions est l'utilisation d'un canon à émission de champ [2] dont les avantages ont été mis en évidence par Crewe [3] et par quelques auteurs ensuite. Au cours de nos expériences il s'est avéré que les cathodes à émission de champ devaient être protégées contre des claquages de la haute tension qui pouvaient les détruire. Une résistance de quelques dizaines de $M \Omega$, introduite en série sur l'arrivée de la haute tension au canon à électrons a remédié à cet inconvénient, mais détruisait par ailleurs la stabilité de la tension d'accélération des électrons à cause des fluctuations du courant d'émission inhérentes au phénomène de l'émission de champ [3,4].

Nous nous sommes donc attachés à améliorer la tension d'accélération de $100 \mathrm{kV}$ fournie par un générateur électrostatique type Samtron 100, dont le taux d'ondulation $\left(1,5 \times 10^{-5}\right)$ est non satisfaisant pour atteindre les hautes résolutions. La méthode

(*) ERA No 376 . utilisée [5], qui ne nécessite aucune intervention sur les circuits électroniques du générateur lui-même, consiste principalement en la réalisation d'un générateur de réaction dont les caractéristiques sont décrites ci-après.

2. Principe de la régulation et diagramme asymptotique de l'affaiblissement. - 2.1 PrINCIPE. - L'ensemble constitué par le générateur $G$ et la résistance de protection $R$ délivre la tension $U+v, v$ étant l'ondulation résiduelle aux bornes de l'utilisation. Une tension d'erreur $\varepsilon$ obtenue à l'aide du diviseur capacitif $C_{1}, C_{2}$, est amplifiée et réinjectée en opposition de phase, en série avec le générateur haute tension $G$, de manière à réduire l'ondulation $v$. Le condensateur $C_{1}$ est constitué par le câble de transport haute tension dont les armatures sont : l'âme centrale du câble qui constitue l'armature haute tension et le blindage qui constitue l'autre armature. Pour éviter toute fluctuation parasite, l'ensemble est blindé à nouveau par rapport à la masse de $G$ et le condensateur $C_{2}$ d'entrée de l'amplificateur de réaction est relié à $C_{1}$

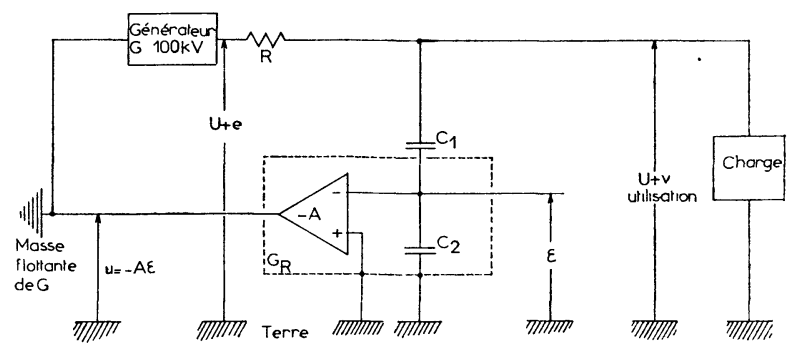

FIG. 1. - Schéma de principe. 
par un câble blindé. La tension d'erreur est alors $\varepsilon=v C_{1} /\left(C_{1}+C_{2}\right)$.

Il est à noter que la résistance $R$, dans le principe, n'est pas utile. Elle a été simplement introduite parce que, dans une utilisation en microscopie électronique à émission de champ, l'expérience nous a montré qu'elle constituait une protection efficace et nécessaire de la cathode contre les claquages de la haute tension. Outre ce rôle de protection, cette résistance présente les avantages suivants :

- Elle permet de reculer le seuil d'apparition d'oscillations lorsqu'on augmente le gain de l'amplificateur en tension. Ces oscillations évidemment détruisent la régulation et apparaissent pour des gains d'autant plus faibles que la résistance $R$ diminue.

- Avec les capacités $C_{1}$ et $C_{2}$ elle constitue un filtre atténuateur des fluctuations de fréquence élevée provenant du générateur $G$.

2.2 Diagramme ASYMPtotiQue DE L'AFFAIBLISSEMENT. - L'ondulation résiduelle $v$ aux bornes de la charge est donnée par l'équation suivante dans le cas où $C_{2}$ est très grand par rapport à $C_{1}$

$$
v=\frac{e}{1+j \omega R C_{1}+\frac{j \omega A R_{2} C_{1}}{1+j \omega R_{2} C_{2}}}
$$

$A$ étant le gain de l'amplificateur de réaction, $e$ l'ondulation, à la fréquence $\omega / 2 \pi$, du générateur haute tension et $R_{2}$ la résistance de fuite du condensateur $C_{2}$ ou l'impédance d'entrée de l'amplificateur.

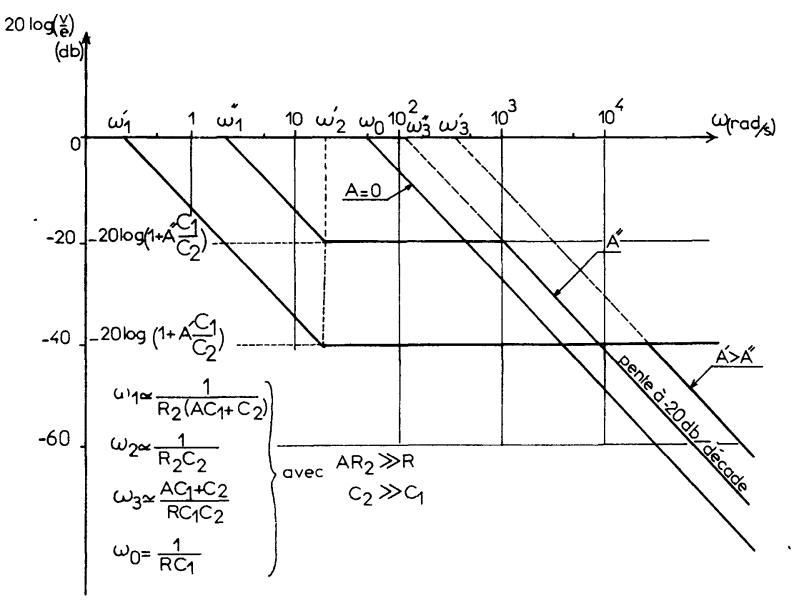

FIG. 2. - Diagramme asymptotique de l'affaiblissement.

Si l'on trace un diagramme asymptotique de l'affaiblissement (Fig. 2) en supposant que $A$ est indépendant de la fréquence, $C_{2} \gg C_{1}$ et $A R_{2} \gg R$, on constate que :

- si le gain $A$ est nul, la résistance $R$ et les capacités $C_{1}$ et $C_{2}$ constituent un filtre atténuateur des fluctuations de pulsation supérieure à $\omega_{0}=1 / R C_{1}$,

- si le gain $A$ est différent de zéro le diagramme pré- sente deux asymptotes coupant l'axe des abscisses à $\omega_{1}=1 / R_{2}\left(A C_{1}+C_{2}\right)$ et à $\omega_{3}=\left(A C_{1}+C_{2}\right) / R C_{1} C_{2}$. Ces asymptotes ont des pentes correspondant à un affaiblissement de $-20 \mathrm{db}$ par décade. Entre ces deux asymptotes l'affaiblissement reste constant sur un palier de fréquence qui est d'autant plus large que $A$ est grand. Il commence à être constant à partir de $\omega_{2}=1 / R_{2} C_{2}$. A gain constant, en augmentant $R_{2}$ et $C_{2}$, on améliorera donc l'affaiblissement des basses fréquences: Quant aux hautes fréquences elles seront affaiblies grâce au filtre $R, C_{1}, C_{2}$.

3. Réalisation pratique. - Le générateur haute tension utilisé est un générateur électrostatique dont l'électronique est à un potentiel flottant par rapport au potentiel de la terre. Les différentes tensions à répartir sur les électrodes du canon à émission de champ sont obtenues à l'aide d'un diviseur résistif $R_{\mathrm{D}}$ plongé dans une cuve remplie d'huile. Le chauffage du filament est commandé par un transistor et une batterie étanche plongés également dans l'huile. Les résistances $R_{1}$ et $R_{0}$ constituent la protection contre des décharges provenant du canon. Des corotrons montés en parallèle entre la première anode et le filament limitent une tension de claquage éventuelle entre ceux-ci à $3,6,9$ ou $12 \mathrm{kV}$ suivant la position du curseur du diviseur résistif. La valeur de la résistance $R_{\mathrm{D}}$ est choisie pour obtenir un débit total du générateur haute tension d'environ $100 \mu \mathrm{A}$ (Fig. 3).

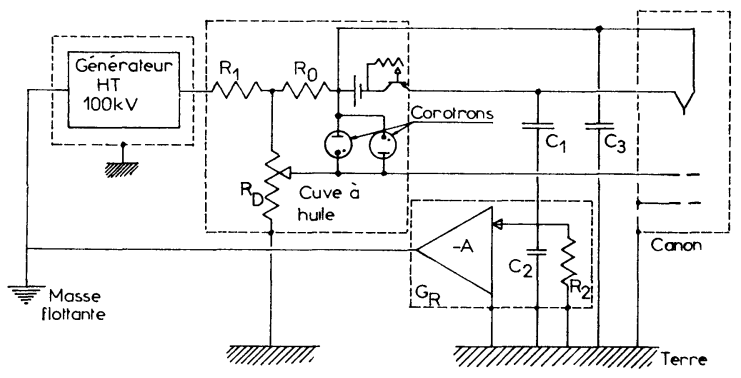

FIG. 3. - Schéma de l'alimentation haute tension du canon à émission de champ.

Le condensateur $C_{1}$ constitue avec $C_{2}$ le diviseur capacitif (cf. paragraphe 2). $C_{3}$ est la capacité entre le blindage du câble haute tension relié à la deuxième borne du filament et son âme centrale. Grâce au filtre $R_{1}, R_{0}, C_{1}, C_{3}$ toutes les fluctuations de haute fréquence provenant du générateur haute tension seront atténuées. Les bruits haute fréquence créés par des décharges dans la cuve à huile ne sont pas filtrés puisqu'ils ont lieu après la résistance $R_{1}$. C'est pourquoi nous avons placé une résistance $R_{0}=3 \mathrm{M} \Omega$ entre le diviseur résistif $R_{\mathrm{D}}$ et le canon qui, avec les capacités $C_{1}$ et $C_{3}$, atténue les fréquences supérieures à $\frac{1}{2} \pi R_{0}\left(C_{1}+C_{3}\right)$ (environ $150 \mathrm{~Hz}$ dans nos conditions expérimentales). Evidemment les bruits au niveau de la commande du chauffage du filament ne sont pas filtrés, et, pour limiter les risques de décharges, une 
attention toute particulière a été portée à l'isolement de cette partie par rapport à la terre.

Les fluctuations de basse fréquence sont compensées par le générateur de réaction $G_{\mathrm{R}}$ qui est constitué de la manière suivante (Fig. 4).

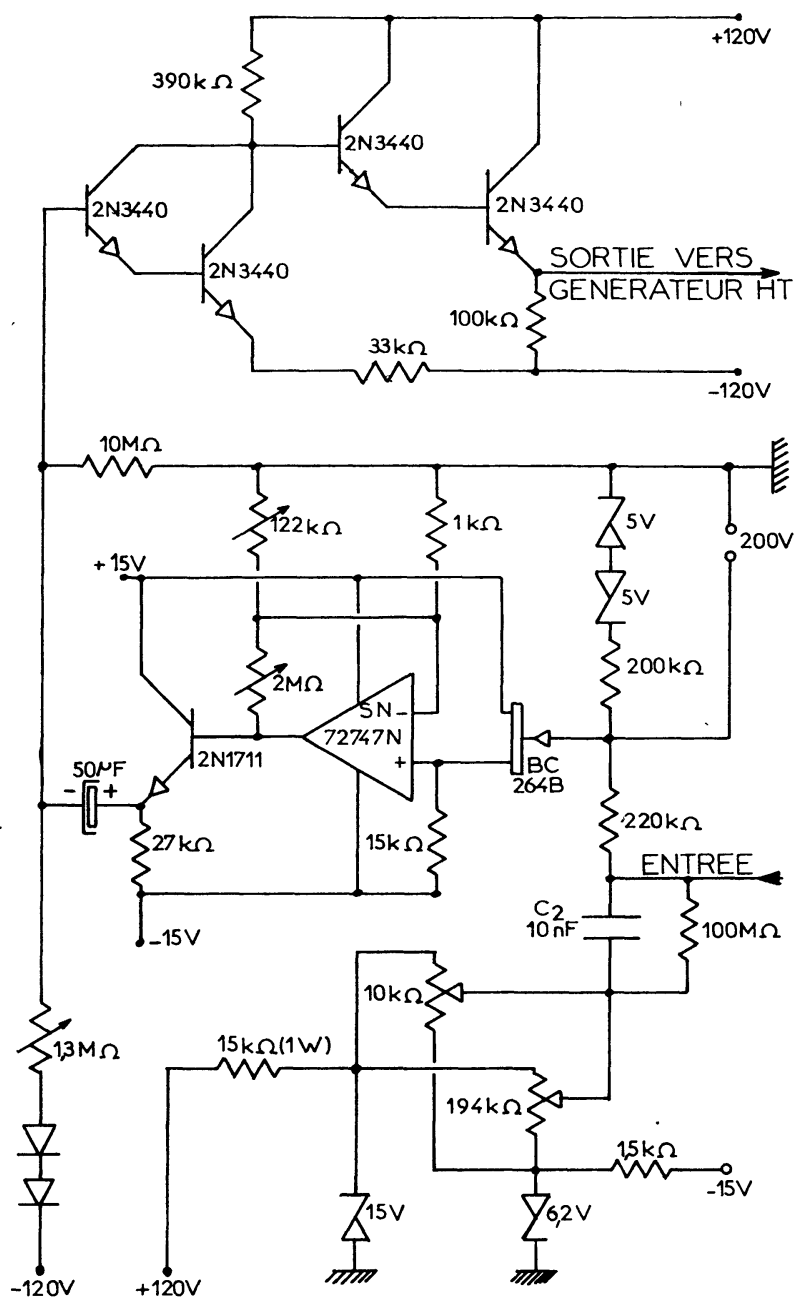

FIg. 4. - Schéma du circuit électronique du générateur de réaction $G_{\mathrm{R}}$.

L'étage d'entrée est un amplificateur opérationnel qui, pour présenter une impédance d'entrée élevée, est précédé par un transistor à effet de champ monté en source commune. Il est protégé en tension par un limiteur à diodes Zener, ainsi que par un éclateur à $200 \mathrm{~V}$. Son gain est réglable de 0 à 2000 et il est relié à un étage de sortie de gain 12 par une liaison capacitive de fréquence de coupure $3 / 1000 \mathrm{~Hz}$; afin d'éviter les dérives il est stabilisé en température. Son impédance de sortie est abaissée à environ $200 \Omega$ grâce à un montage Darlington monté en émetteur commun.

Les résistances de fuite des condensateurs $C_{1}$ et $C_{2}$ constituent un pont résistif qui peut amener sur l'entrée de l'amplificateur d'erreur une tension de plusieurs volts et donc saturer cet amplificateur. Un réglage de tension de décalage à l'entrée est donc prévu pour annuler cette tension.
4. Résultats expérimentaux. - 4.1 EN MICROSCOPIE CONVENTIONNELle. - Sur les microscopes conventionnels des cathodes à chauffage thermoionique sont utilisées. Celles-ci délivrent un courant électronique suffisamment constant dans le temps pour que nous assimilions cette utilisation à celle d'un générateur haute tension débitant sur une charge constante. Evidemment notre système de régulation n'est pas restreint à la microscopie mais s'applique chaque fois que la charge sera non fluctuante.

Nous avons étalonné à l'aide d'un générateur basse tension variable en fréquence l'atténuation due au filtre $R, C$ seul (Fig. 5).

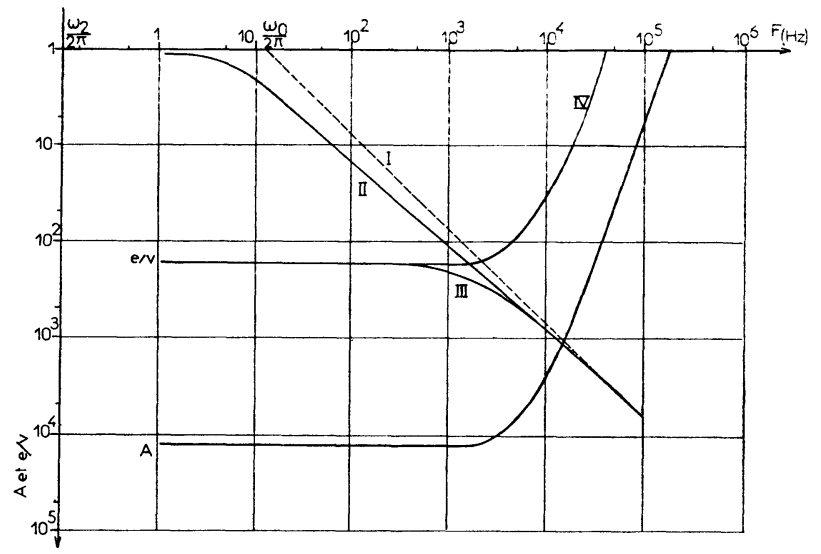

Fig. 5. - Courbes expérimentales, en fonction de la fréquence, du gain $A$ du générateur de réaction et du facteur d'atténuation $e / v$. Les courbes I et II représentent respectivement l'atténuation théorique et expérimentale du filtre $R, C$ seul ( $A$ est nul). La courbe III donne le facteur d'atténuation de l'ensemble générateur de réaction plus filtre $R, C$ quand $R=100 \mathrm{M} \Omega$ et quand $A$ a les valeurs ci-dessus. La courbe IV donne le facteur d'atténuation lorsqu'on ne tient pas compte de l'effet du filtre $R, C$, c'est-à-dire qu'elle donne l'atténuation des fluctuations qui proviennent directement de la charge.

Puis ensuite nous avons mesuré l'amplitude de la tension $u_{1}$ à la sortie du générateur de réaction $G_{\mathrm{R}}$ quand celui-ci est connecté à la masse flottante du générateur et l'amplitude $u_{2}$ quand il est connecté à la terre. Nous avons :

$$
u_{1}=A v_{1} \frac{C_{1}}{C_{1}+C_{2}} \quad \text { et } \quad u_{2}=A v_{2} \frac{C_{1}}{C_{1}+C_{2}}
$$

Le rapport $u_{2} / u_{1}=v_{2} / v_{1}$ multiplié par l'atténuation propre du filtre $R, C$ nous donne le facteur d'atténuation $e / v$ de l'ensemble générateur de réaction et filtre $R, C$ (Fig. 5).

Cet étalonnage a été effectué pour une certaine valeur du gain $A=u_{2} / \varepsilon$,

$$
\varepsilon=v_{2} \frac{C_{1}}{C_{1}+C_{2}} \text {. }
$$

Sur la figure 5 on constate que le gain est constant de $1,5 \mathrm{~Hz}$ à $2000 \mathrm{~Hz}$ (nous n'avons pas effectué de 
mesures en dessous de $1,5 \mathrm{~Hz}$ ), puis décroît pour les fréquences supérieures et l'effet du générateur de réaction est pratiquement nul aux fréquences supérieures à quelques dizaines de milliers d'Hertz.

Pour un réglage du gain de l'amplificateur de réaction correspondant à la figure 5 , nous avons mesuré l'amélioration du système sur notre générateur haute tension. Celui-ci ayant une ondulation résiduelle de fréquence $100 \mathrm{~Hz}$ et d'amplitude $1,5 \mathrm{~V}$ son taux d'ondulation propre à $100 \mathrm{kV}$ est $1,5 \times 10^{-5}$. Pour un gain $A=12000$ à cette fréquence, le facteur d'atténuation mesuré est environ 180 , ce qui donne donc un taux d'ondulation aux bornes de l'utilisation égal à $8 \times 10^{-8}$.

Notons qu'il nous a été possible de travailler à un gain légèrement supérieur à 12000 , mais pratiquement nous nous sommes limités à cette valeur pour être sûr d'éviter l'apparition d'oscillations qui rendent le système inefficace.

4.2 EN MICROSCOPIE A ÉMISSION DE CHAMP. Le courant électronique donné par les cathodes à émission de champ fluctue [3-4] et il est équivalent de dire que la charge d'utilisation fluctue dans le temps puisque la tension d'accélération est constante.

Ces fluctuations de courant d'émission créent aux bornes de la résistance de protection $R=R_{1}+R_{0}$ (Fig. 3) une fluctuation de tension qui détruit la stabilité de la tension d'accélération. Le générateur de réaction qui stabilise la tension directement aux bornes de l'utilisation se justifie donc particulièrement dans cette utilisation.

L. H. Veneklasen [4] a donné l'amplitude des instabilités du courant d'émission en fonction de leur fréquence. Cette amplitude est maximum autour de la fréquence $100 \mathrm{~Hz}$, fréquence pour laquelle notre système réagit particulièrement bien.

Il est à noter que, pour les instabilités provenant de la charge, le filtre $R, C$ n'a aucun rôle dans l'affaiblissement. Dans ce cas, le facteur d'atténuation décroît pour les fréquences supérieures à $2000 \mathrm{~Hz}$ et le système devient complètement inefficace au-dessus de $40000 \mathrm{~Hz}$. Ceci n'est pas gênant car l'amplitude des fluctuations du courant d'émission (pour une valeur raisonnable de celui-ci. Voir ci-dessous) est très faible [4].

Nos mesures ont été effectuées de la manière suivante : tout d'abord nous avons mesuré l'amplitude $u_{1}$ quand la cathode à émission de champ ne débite pas, c'est-à-dire quand nous sommes dans le cas d'une utilisation sur charge constante (cf. paragraphe 4.1). Nous avons vu que dans ce cas le taux d'ondulation est $8 \times 10^{-8}$. Ensuite nous avons mesuré l'amplitude $u_{1}$ à la sortie du générateur de réaction quand le canon à émission de champ fonctionne et que le courant d'émission est $5 \mu \mathrm{A}$. Avec une cathode à la température de la salle, les contaminants ayant été expulsés préalablement en portant cette cathode à $2000{ }^{\circ} \mathrm{C}$ pendant quelques secondes, nous avons obtenu une amplitude $u_{1} 4$ fois plus grande que lorsque la charge est constante. On en déduit donc que le taux d'ondulation à $100 \mathrm{kV}$ est de l'ordre de $3 \times 10^{-7}$. Pour le même courant d'émission, mais avec une cathode chauffée à environ $1000^{\circ} \mathrm{C}$, les fluctuations du courant d'émission sont plus importantes et l'amplitude $u_{1}$ dans ce cas est environ 20 fois plus élevée que lorsque la charge est constante, ce qui donne un taux d'ondulation de l'ordre de $1,5 \times 10^{-6}$. Dans nos expériences la pression dans le canon était égale à $1 \times 10^{-9}$ torr. Nous avons également constaté que le taux d'ondulation restait inférieur à $1 \times 10^{-7}$ lorsque le courant d'émission est $1 \mu \mathrm{A}$. Ceci est normal puisque l'amplitude des fluctuations du courant d'émission est à peu près proportionnelle à la valeur de celui-ci. On constate donc que le taux d'ondulation de la tension d'accélération des électrons dépend étroitement de la valeur du courant d'émission, mais aussi de la résistance de protection $R$. Pour une valeur du courant d'émission de $5 \mu \mathrm{A}$ qui donne une brillance du faisceau électronique très élevée de 1 à $5 \times 10^{8} \mathrm{~A} / \mathrm{cm}^{2} \times$ stéradian à $100 \mathrm{kV}[6,7]$, le taux d'ondulation est encore de l'ordre de $1 \times 10^{-6}$. On pourra améliorer celui-ci en réduisant la valeur du courant d'émission si l'on peut se contenter d'une brillance plus faible. On pourra aussi facilement l'améliorer en réduisant la valeur de la résistance de protection $R$, mais ceci en augmentant les risques de destruction de la pointe par une décharge. L'idéal est d'avoir une résistance $R$ réglable qu'on adapte aux qualités de tenue en tension du canon.

Remarquons que cette résistance $R$ peut aussi créer une dérive de la tension d'accélération à cause des dérives du courant d'émission. Il a été montré récemment [1] que des dérives linéaires de la haute tension ne sont pas une sérieuse limitation de la résolution. Une dérive linéaire de $5 \times 10^{-7}$ pendant le temps de pose de la photographie (quelques secondes tout au plus) permet des résolutions de $1 \AA$. Ceci correspond donc à des alimentations haute tension dont la dérive serait de l'ordre de $1 \times 10^{-5} / \mathrm{min}$, valeur facilement obtenue avec les alimentations des microscopes commerciaux. Néanmoins, si des dérives de cet ordre ne limitent pas la résolution elles sont très 'gênantes dans les observations de longue durée et mises au point de l'image électronique. En aucuń cas donc, des dérives supérieures ne sont acceptables or ceci peut se produire pour certaines utilisations du canon à émission de champ requérant une brillance élevée. En effet, une telle brillance nécessite un courant d'émission assez important et la dérive de celui-ci, de part la grandeur de la résistance $R$, peut entraîner une dérive de la tension d'accélération supérieure à cette valeur de $1 \times 10^{-5} / \mathrm{min}$.

Pour y remédier il suffirait tout simplement que le courant d'émission ne circule pas à travers la résistance $R$. Dans ce but nous envisageons de remplacer le diviseur résistif (Fig. 3) par une alimentation $10 \mathrm{kV}$ dont la borne négative est reliée au filament et la borne positive à la première anode. De cette manière la 
presque-totalité du courant d'émission est fournie par cette alimentation et de ce fait, le courant de faisceau électronique (quelque $10^{-8} \mathrm{~A}$ ), qui traverse la résistance de protection $R$, ne contribue à la dérive que pour une part négligeable.

5. Conclusion. - Grâce à un montage électronique nous améliorons le taux d'ondulation de la tension accélératrice des électrons d'un canon à émission de champ. Celui-ci, primitivement égal à $1,5 \times 10^{-5}$ à $100 \mathrm{kV}$ est divisé par un facteur variant de 10 à 100 suivant les conditions d'utilisation. Nous espérons qu'assez de détails ont été donnés pour permettre à d'autres utilisateurs, ayant des problèmes similaires aux nôtres, d'améliorer facilement le taux d'ondulation de leur générateur haute tension.

\section{Bibliographie}

[1] Beorchia, A. et Bonhomme, P., Optik (à paraître).

[2] Troyon, M., Thèse $3^{\mathrm{e}}$ cycle. Fac. Sciences de Reims (juillet 1971).

[3] Crewe, A. V., Eggengerger, D. N., Wall, J. and Welter L. M., Rev. Sci. Instrum. 39 (1968) 576.

[4] Veneklasen, L. H., Ph. D. Thesis, Correl University
Ithaca, New York (1971) Materials Sciences Center Report $\mathrm{n}^{\circ} 1525$

[5] GIRARD, M., Elect. Letters, 8 (1972).

[6] Veneklasen, L. and Siegel, B. M., J. Appl. Phys. 43 (1972), 4989.

[7] Troyon, M., Kuo, H. P., et Siegel, B. M., Thirty-first annual E. M. S. A. Meeting (août 1973). 